\title{
An Atypical Case of Hepatosplenic T Cell Lymphoma
}

\begin{abstract}
Hepatosplenic T cell lymphoma (HSTL) is a rare, aggressive subtype of extra-nodal lymphoma characterized by hepatosplenic presentation without lymphadenopathy that is typically seen in adolescents and young adults with a male predominance with bone marrow involvement. We present a case of a 55 year old woman with past history of pulmonary embolism, deep venous thrombosis and type II diabetes who complained of diffuse abdominal pain (worst in the LUQ) and lightheadedness. Ultimately, the patient was diagnosed with splenic hematoma and hemoperitoneum. This diagnosis necessitated splenectomy which revealed a markedly enlarged and ruptured spleen. Microscopic analysis with immunohistochemical stains showed a prominent abnormal T-cell population involving the splenic red pulp consistent with HSTL in this patient. Follow-up bone marrow biopsy was negative for involvement. We present this patient's case due to her unique demographic and the atypical clinical features of her disease including lack of bone marrow presentation.
\end{abstract}

\section{Introduction}

Hepatosplenic T cell lymphoma is a rare, aggressive subtype of T-cell non-Hodgkin lymphoma with extra-nodal and systemic presentations [1]. This neoplasm usually derives from cytotoxic $\mathrm{T}$ cells expressing a gamma-delta receptor type [2]. Though the etiology of the disease is unknown, as much as $20 \%$ of HSTL cases arise in the setting of chronic immune suppression [3]. Clinical findings include hepatomegaly, splenomegaly, systemic symptoms, anemia, thrombocytopenia, leukopenia, and liver enzyme abnormalities in the absence of lymphadenopathy [4]. The disease predominately affects young males (20-35 years of age) and has a rapidly progressive course with mean overall survival of less than 16 mos. regardless of treatment [5].

We present an atypical case of HSTL both in demographic and clinical course. Our patient, a 55 year old female with past history of pulmonary embolism, deep venous thrombosis, and diffuse abdominal pain, presented from an outside hospital with a immediate history of traumatic splenic rupture and hemoperitoneum, necessitating splenectomy. Architectural distortion noted on microscopic analysis of splenic tissue as well as follow up immunohistochemistry revealed an underlying diagnosis of HSTL. While reports of unusual HSTL cases exist in the literature, this case is one that is granzyme negative and lacks bone marrow involvement. Analyzing pathophysiology in light of unusual case presentations can improve understanding of a disease and the most appropriate treatment option.

\section{Case Description}

A 55 year old female with a past history of pulmonary embolism, deep venous thrombosis, and type II diabetes presented to the emergency department upon referral from an outside facility with lightheadedness and diffuse, worsening abdominal pain accompanied by nausea and vomiting with concern for traumatic splenic rupture. At the referring institution, the patient was noted to have a splenic
Journal of

Hematology \& Thrombosis

\author{
Gosnell $\mathrm{HL}^{1 *}$ and Stump MS ${ }^{123}$

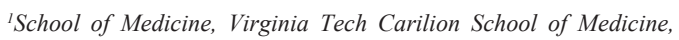 \\ USA \\ ${ }^{2}$ Section of Pathology, Virginia Tech Carilion School of Medicine, \\ USA \\ ${ }^{3}$ Dominion Pathology Associates, USA
}

\section{*Address for Correspondence:}

Gosnell HL, School of Medicine, Virginia Tech Carilion School of Medicine, 1 Riverside Circle, Suite 105, Roanoke, Virginia 24016, Virginia, USA, Phone: 252-241-4428; E-mail: haileylg@vt.edu

\section{Submission: 07 July, 2020 \\ Accepted: 19 August, 2020}

Published: 21 August, 2020

Copyright: ๑ 2020 Gosnell HL, et al. This is an open access article distributed under the Creative Commons Attribution License, which permits unrestricted use, distribution, and reproduction in any medium, provided the original work is properly cited.

hematoma and hemoperitoneum on CT as well as a hemoglobin value of $7.5 \mathrm{~g} / \mathrm{dL}$ and marked hypotension (systolic blood pressure measured in the 80s). The patient was given $\mathrm{K}$ centra and three liters of fluid on transport to our facility (Figure 1).

On physical exam, the patient was noted to be hypotensive with sinus tachycardia (100s), pale conjunctivae, diffuse abdominal tenderness (most severe in the LUQ), and dry, pale skin. No external signs of injury were noted. FAST exam confirmed a large amount of abdominal free fluid. The patient was noted to have acute blood loss anemia likely from either splenic hematoma or splenic artery aneurysm. One liter of blood was promptly transfused and a splenectomy was performed.

Intraoperatively the spleen was noted to be essentially in pieces with clotted blood. Microscopically the white pulp appeared intact but infringed upon by an expanded red pulp containing a lymphoid process sinuses and cords. These cells consisted of medium-sized, atypical lymphocytes, exhibiting irregular nuclear borders and clear cytoplasm. Background histiocytes exhibiting hemophagocytosis as well as scattered background lymphocytes and immunoblasts were noted. Review of the gross description, provided by a rotating pathologist assistant student, revealed the spleen weighed 862 grams (Figure 2).

Suspecting an underlying hematologic malignancy, immunohistochemical stains were performed and the initial battery

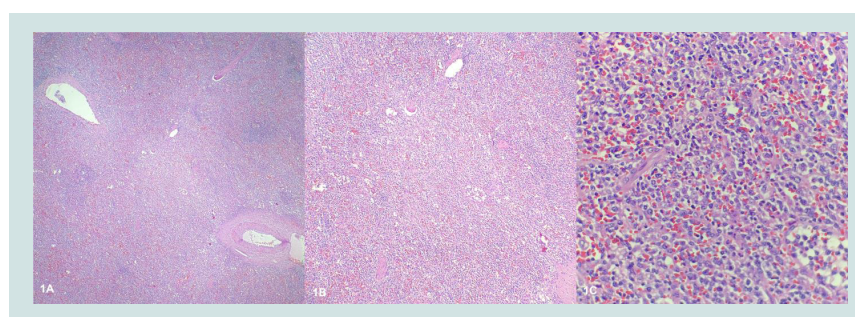

Figure 1: (The H\&Es): (1A and $1 \mathrm{~B})$ expanded red pulp (taken at $5 \mathrm{x}),(1 \mathrm{C})$ Increased irregular mononuclear cells within red pulp (taken at 40x). 


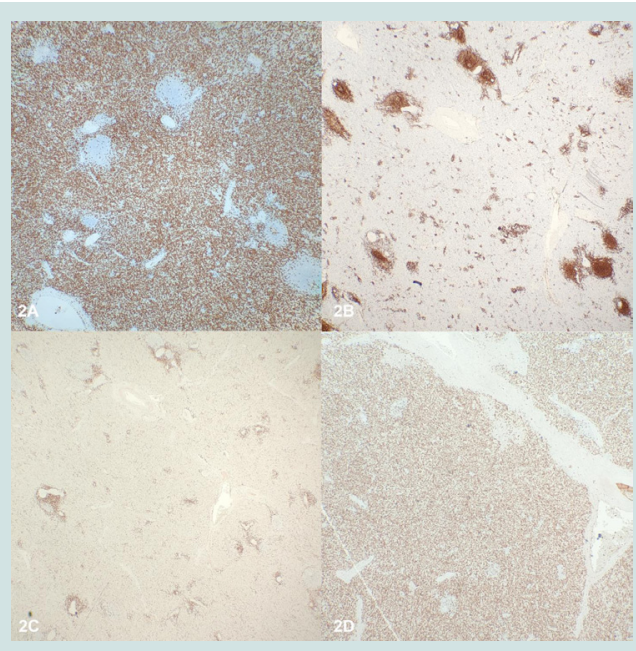

Figure 2: CD3 showing expanded T-cell population within red pulp (2A) and CD20 showing B-cells confined mostly to white pulp (2B). Loss of CD5 within T-cells (2C) while retaining CD7 (2D).

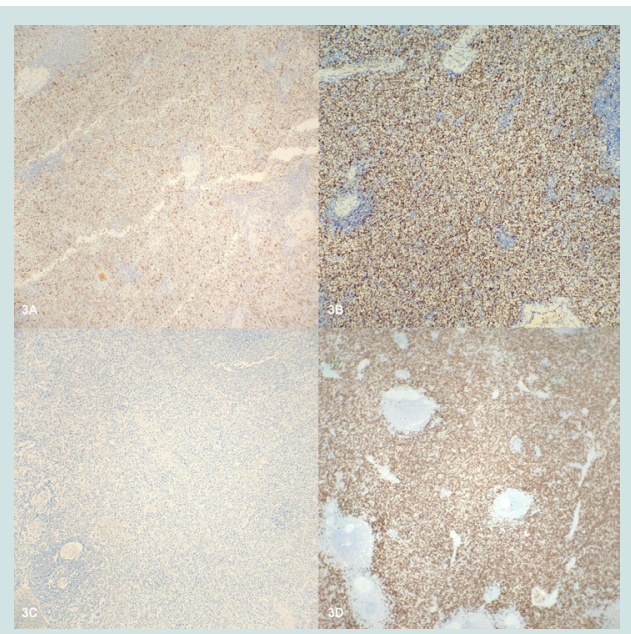

Figure 3: Neoplastic T-cells express Perforin (3A) and TIA-1 (3B) and are negative for TCR-BetaF1 (3C) while expressing TCR-Delta (3D).

identified an expanded T-cell population within the red pulp that expressed CD3 and CD7 but was negative for CD5. Full workup revealed this population expressed $\mathrm{CD} 3$, subset $\mathrm{CD} 8, \mathrm{CD} 7$, TIA1, perforin, minimal CD56 and TCR delta while being negative for CD4, CD5, TCRBF1, granzyme B, CD30, CD57, CD34, CD99, TdT, EBER ISH and B-cell markers. PCR studies identified TCRG and TCRB rearrangements consistent with a clonal T-cell population. Unfortunately, flow cytometry was not performed. There was no evidence of lymphadenopathy and review of the peripheral smear from an admission specimen did not show an abnormal leukocyte population. Given these findings a diagnosis of hepatosplenic T-cell lymphoma was made and this case was sent for second opinion to University of Virginia Health System with agreement of that diagnosis.

A follow-up bone marrow biopsy, performed at an outside hospital, showed hypercellular marrow with mild CD3+ T cell lymphocytosis without abnormal immunophenotype. Flow cytometry of the bone marrow also did not identify a T-cell lymphoproliferative process. The patient's bone marrow karyotype was found to be normal. FISH specifically looking for isochromosome $7 \mathrm{q}$ was negative. Cancer gene mutation panel did not reveal any CML or myeloproliferative neoplasm related DNA mutations or RNA translocations (Figure 3).

\section{Discussion}

We presented a unique case of HSTL lacking bone marrow involvement in a 55 year old female. The patient's hematologic malignancy was not readily apparent until histological analysis was performed post splenectomy (indicated for perceived splenic hematoma and hemoperitoneum). While flow cytometry was not performed, the morphology and judicious use of immunohistochemical stains provided key information in support of a HSTL diagnosis.

The patient's demographic and clinical course made solidifying a case for an HSTL diagnosis challenging. As aforesaid, hepatosplenic $\mathrm{T}$ cell lymphoma namely affects young males and our patient is a middle-aged female. In addition, the disease almost always shows some degree of bone marrow involvement [6,7]. Our patient's clinical picture did not reflect bone marrow involvement. Our patient's atypical $\mathrm{T}$ cells were negative for granzymes $\mathrm{B}$ and $\mathrm{M}$, and positive for perforin. The atypical $\mathrm{T}$ cells of HSTL patients usually express granzyme B and fail to express perforin [5,8]. Amid the aspects of this patient's case that failed to conform with a textbook case of HSTL, there were a number of factors, taken together, made this diagnosis the most likely.

Despite an atypical demographic and case presentation, the diagnosis of HSTL was chosen for this patient in light of splenic histology, T cell markers, and PCR studies. On microscopic analysis, our patient's splenic white pulp appeared intact but infringed upon by red pulp sinuses and cords consisting of medium, atypical lymphocytes exhibiting irregular nuclear borders and clear cytoplasm. The splenic histology noted in this description is consistent with that described in a typical HSTL case (i.e. neoplastic cells surrounded by rims pale cytoplasm infiltrating the cords and sinuses of splenic red pulp with atrophy of white pulp) [9]. Like those of a typical HSTL case, the patient's $\mathrm{T}$ cells displayed a gamma-delta immunophenotype, with rearranged TCRG and TCRB genes noted on PCR.

Within the differential diagnosis, an atypical large granular $\mathrm{T}$ cell Leukemia/Lymphoma (T-LGL) was considered in light of the patient's demographic, clinical course, and lab results. Our patient's demographic is more in line with that of a typical case of T-LGL (equal male to female ratio affected with majority of cases presenting between 45-75 years of age) [10]. The lack of bone marrow involvement noted in our patient's case is also made more plausible by a T-LGL diagnosis, as bone marrow involvement is variable in T-LGL [11].

A number of core features of this patient's case including splenic histology, lack of abnormal lymphocyte population on peripheral smear, and the immunophenotype, did not match up with a T-LGL diagnosis, however. Microscopic splenic findings in a typical case of T-LGL show infiltration and expansion of the red pulp cords and sinusoids by T-LGLs with sparing of the often hyperplastic white pulp7 a description not consistent with the splenic architecture noted in 
our patient's case. Additionally, the atypical T-cells in T-LGL usually display a CD57+, granzyme $\mathrm{B}+$, alpha/beta+ immunophenotype, with only rare cases expressing gamma/delta [12]. Finally, there was no peripheral blood involvement or even a lymphocytosis at the time of splenectomy or bone marrow biopsy that would be expected in T-LGLL.

\section{Conclusion}

Our case illustrates and unusual presentation of HSTL with spontaneous splenic rupture in a patient that does not fit the characteristic demographic. The lack of bone marrow involvement is also unusual compared to cases reported in the literature. This case also highlights the need for a pathologist to have a high degree of suspicion and a low threshold for performing stains to exclude neoplastic processes in cases of splenomegaly that may not have abundant history or is otherwise unexplained. Furthermore, an extensive immunohistochemical workup and wisely reviewing all available material present can usually yield an accurate diagnosis despite not having all possible testing modalities available.

\section{References}

1. Ferreri AJ, Govi S, Pileri SA (2012) Hepatosplenic gamma-delta T cell lymphoma. Crit Rev Oncol Hematol 83: 283-292.

2. Rosh JR, Gross T, P Mamula, A Griffiths, J Hyams (2007) Hepatosplenic T cell lymphoma in adolescents and young adults with Crohn's disease: a cautionary tale? Inflamm Bowel Dis 13: 1024-1030.

3. Weidmann E (2000) Hepatosplenic T cell lymphoma: A review on 45 cases since the first report describing the disease as a distinct lymphoma entity in 1990. Leukemia 14: 991-997.
4. Belhadj M, Mansour D, Kaltenbach S, Deau-Fischer B, Franchi P, et al. (2019) $T$ cell large granular lymphocyte leukemia transformation into aggressive T-cell lymphoma: a report of two cases with molecular characterization. Haematologica 104: e117-e120.

5. Shaw GR, Naik VS (2008) The gammadelta variant of T cell large granular lymphocyte leukemia is very similar to the common alpha-beta type: report of two cases. J Hematop 1: 139-143.

6. Swerdlow SH, Campo E, Harris NL, ES Jaffe, SA Pileri, et al. (2008) World Health Organization classification of tumours of haematopoietic and lymphoid tissues ( $4^{\text {th }}$ Edn). Int Agency for Research on Cancer press 2.

7. Alsohaibani FI, Abdulla MA, Fagih MM (2011) Hepatosplenic T cell lymphoma. Indian Journal of Endocrinology and Metabolism 27: 39-42.

8. Gaulard P, Jaffe ES, L Krenacs, WR Macon (2008) Hepatosplenic T cell lymphoma. WHO Classification of tumours of haematopoetic and lymphoid tissues $381-382$.

9. Macon WR, Levy N, Kurtin PJ, Salhany KE, Elkhalifa MY, et al. (2001) Hepatosplenic ab T-cell lymphomas: a report of 14 cases and comparison with hepatosplenic cd T-cell lymphomas. Am J Surg Pathol 25: 285-296.

10. Madabhavi I, Modi G, Panchal H, Patel A, Revannasiddaiah S, et al. (2017) Hepatosplenic Gamma Delta T Cell Lymphoma: Two Rare Case Reports from Western India. Int J Hematol Oncol Stem Cell Res 11: 296-300.

11. Osuji N, Matutes E, Catovsky D, Lampert I, Wotherspoon A (2005) Histopathology of the spleen in T cell large granular lymphocyte leukemia and T cell prolymphocytic leukemia: a comparative review. Am J Surg Pathol 29: 935-941.

12. Suarez F, Wlodarska I, Rigal-Huguet F, Mempel M, Martin-Garcia N, et al. (2000) Hepatosplenic ab T-cell lymphoma: an unusual case with clinical, histologic, and cytopgenietic features of cd hepatosplenic T-cell lymphoma. Am J Surg Pathol 24: 1027-1032. 